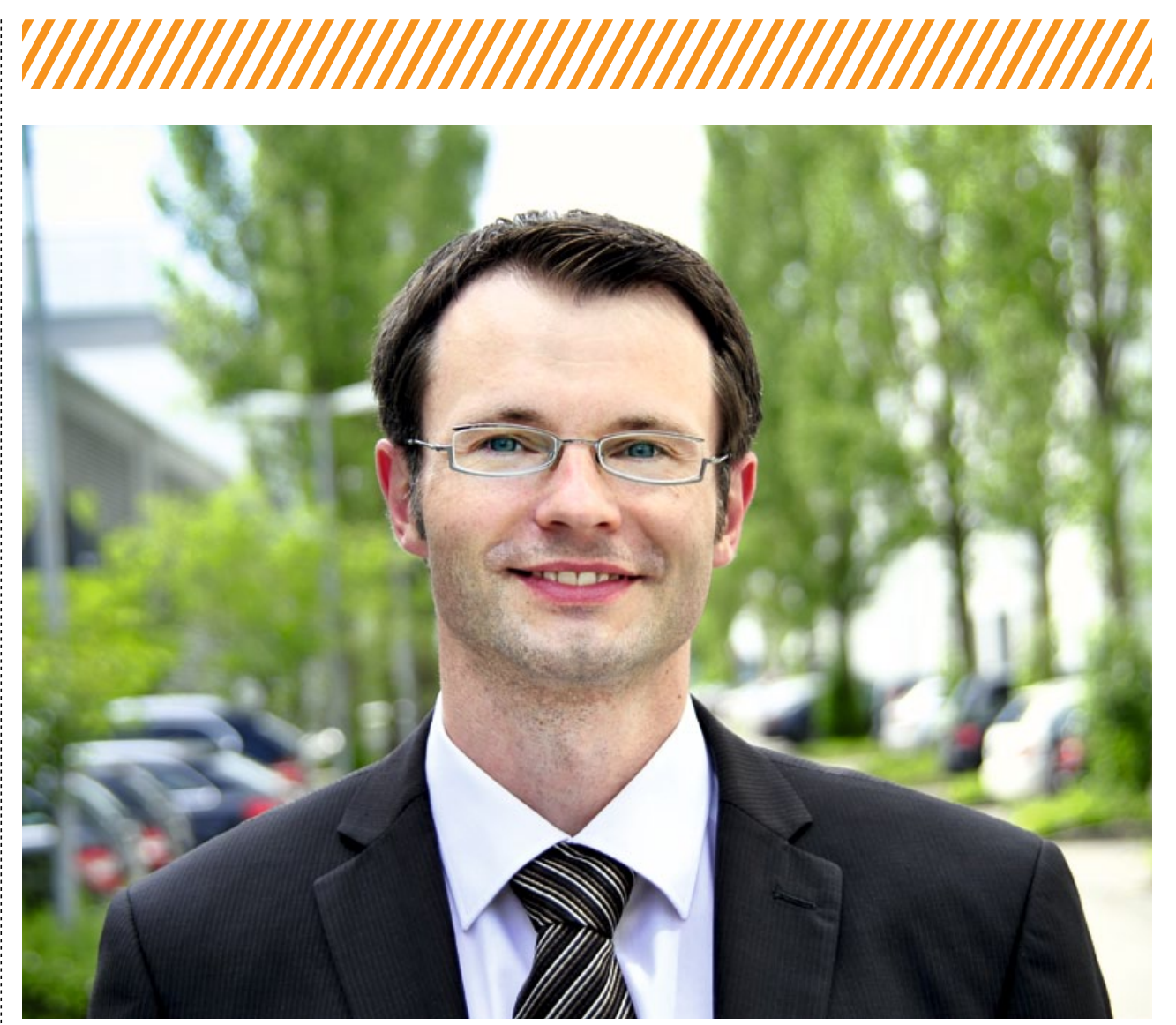

\section{UDO HORNFECK}

Leiter Technologie- und Innovationsmanagement sowie Entwicklungsleiter Elektrikkomponenten, DräxImaier Group

\title{
PARADIGMENWECHSEL IM BORDNETZ
}

Fahrerassistenzfunktionen für das hochautomatisierte Fahren werden die Anforderungen an das Bordnetz und damit auch einige Paradigmen nachhaltig verändern. Dabei spielt der Aspekt der funktionalen Sicherheit eine entscheidende Rolle. Traditionelle sicherheitskritische Funktionen wie Lenk- oder Bremsunterstützung werden größtenteils mithilfe mechanischer Redundanzen abgesichert, was bei automatisierten Fahrfunktionen prinzipiell nicht mehr möglich ist. Versorgungssysteme haben derzeit im Sinne der Sicherheit das vorrangige Ziel, Leitungsbrände zu verhindern, und schalten im Fehlerfall in einen sicheren Zustand, auch Fail-safe genannt. Hier muss der gravierendste Wandel stattfinden, hin zu Fail-operationalSystemen, die auch im Fehlerfall wichtige Teilnetze für eine definierte Zeitspanne aufrechterhalten können.

Wie können künftige Lösungen nun aussehen? Das Energiebordnetz wird sich zukünftig stark verändern. Für die Umsetzung intelligenter Steueralgorithmen werden Software und Elektronik an die Stelle von Relais und Schmelzsicherungen treten. Zuverlässigkeit muss dann neu kalkuliert werden. Jahrelang konnte auf den „Stand der Technik“ verwiesen werden, da sich die Bauteile in der Praxis bewährt hatten. Für neue Technologien dagegen ist die Zuverlässigkeit nach ISO 26262 zunächst sehr aufwendig nachzuweisen.
Aus Systemsicht stellt sich die Frage, welche Rolle der Energieversorgung im Sicherheitskonzept zukommt? Werden weiterhin Funktionen separat betrachtet, oder gibt es zukünftig eine funktional sichere Energieversorgung, auf die sich alle Funktionen abstützen können? Jeder elektrische Verbraucher definiert dabei seine Anforderungen an die Energieversorgung hinsichtlich Verfügbarkeit, damit ein System namens Safety-EnergyManager entsprechend Prioritäten nach Sicherheitsstufen oder Relevanz für den Fahrer vergeben kann. Elektrische Verbraucher mutieren dabei zu einer Zwei- oder Dreiklassengesellschaft. Teilnetze sowie notwendige Redundanzen im Bereich Speicher, Wandler, Verbindungen, Sensorik/Aktorik oder Halbleiter können partitioniert und verschiedene Spannungsebenen für das Konzept einer Notstromversorgung kombiniert werden.

Zukünftige Innovationen werden den Wandel der elektrischen Energieversorgung im Bordnetz stark beschleunigen. Dabei gibt es noch einige offene Fragen im Bereich der Systemarchitektur oder der Bewertung der Zuverlässigkeit, bei denen eine gemeinsame Sichtweise der Automobilindustrie sehr wünschenswert ist. Die Entwicklung intelligenter Bordnetzlösungen hat letztendlich das hauptsächliche Ziel, neue, anspruchsvolle Funktionen zu ermöglichen und somit die Innovationskraft der Automobilindustrie im Bereich elektrischer Funktionen zu erhalten. 